\title{
THE MINIMAL BOUNDARY OF $C(X)$
}

\author{
BY \\ S. L. GULICK( $\left.{ }^{1}\right)$
}

1. Introduction. Let $X$ be a nonvoid completely regular Hausdorff space, let $C(X)$ be the space of all continuous, complex-valued functions on $X$, and let $E \subseteq C(X)$ be a subspace which separates points of $X$ and contains the constant functions and is an algebra under pointwise multiplication. In [1] Bishop proved that if $X$ is compact and metrizable, and if as a subspace of $C(X)$ with the supremum norm, $E$ is a Banach algebra, then there exists a smallest subset $M$ of $X$ with the property that each function in $E$ takes on its maximum absolute value on $M$. He called $M$ the minimal boundary and exhibited a few of its properties. Bishop also showed that if $X$ was compact but not metrizable, then the minimal boundary need not exist.

In $\S 2$ we remove the restriction of metrizability on $X$, and consider spaces of type $C(X)$. We say that $C(X)$ (or sometimes $X$ ) has a minimal boundary $M_{X}$ if $M_{X}$ is the smallest subset of $X$ with the property that each function which is bounded and attains its maximum absolute value on $X$ in fact attains this maximum absolute value on $M_{X}$. In our fundamental Theorem 2.7 we characterize those spaces $X$ for which $M_{X}$ exists. As a consequence we observe that if $M_{X}$ does exist, it is dense in $X$ and it consists precisely of all the $G_{\delta}$ points in $X$. If $X$ is metrizable or dispersed, then $M_{X}$ always exists, while if $X$ is an infinite compact extremally disconnected space, then $M_{X}$ cannot exist. We complete the section by giving an example in which the minimal boundary $M_{X}$ exists, but is not even a Borel subset of $X$.

$\S 3$ deals with inheritance properties of minimal boundaries with respect to subspaces and products of given spaces. The main theorem of the section, Theorem 3.1, says the following. An arbitrary completely regular Hausdorff space $Y$ can be embedded topologically in a completely regular Hausdorff space $X$ such that $M_{X}$ exists and such that if $M_{Y}$ exists, then $M_{Y}=M_{X} \cap Y$. It follows from this theorem that if $X$ is such that $M_{X}$ exists, then it is by no means true that for each closed subspace $Y$ of $X, M_{Y}$ must exist. However, it is true for any subspace $Y$ which is a $G_{\delta}$ (closed or not) in $X$. Finally we show that countable products of spaces with minimal boundaries also have minimal boundaries, while uncountable products of nontrivial spaces never have minimal boundaries.

$\S 4$ concludes the paper. In it we demonstrate that if $X$ is not compact, then the

Presented to the Society, January 28, 1967; received by the editors November 7, 1966.

(1) This research was almost completely supported by the National Science Foundation Grant GP-6157. 
Stone-Čech compactification $\beta X$ of $X$ has a minimal boundary if and only if $X$ is pseudo-compact and $X$ itself has a minimal boundary. From this result we deduce that $C\left(M_{X}\right)$ has a natural identification with $C(X)$ only when $M_{X}$ is pseudocompact and $\beta M_{X}=X$.

The author acknowledges with pleasure discussions with Professors William G. Bade and Adam Kleppner.

2. Characterization of the minimal boundary. We set about to characterize the minimal boundary of $C(X)$, and list a few of its properties and consider several examples.

2.1. Definition. Let $X$ be an arbitrary topological space. Let $f$ be a real-valued continuous function on $X$. Let

$$
B_{f}=\{x \in X:|f(x)|=\sup \{|f(y)|: y \in X\}\} .
$$

Let $C_{s}(X)$ consist of all continuous, real-valued functions $f$ on $X$ such that $B_{;} \neq \varnothing$.

Then $f \in C_{s}(X)$ if and only if $f$ attains its maximum absolute value on $X$. Note that $C_{s}(X)=C(X)$ if and only if $X$ is pseudo-compact. Furthermore, $C_{s}(X)$ need not be closed under multiplication of functions. For let $X=[-1,1)$ and let

$$
\begin{aligned}
f(x)=|x|, \quad x \in X, \quad g(x) & =\frac{1}{2} x+\frac{1}{2}, & & x<0, \\
& =\frac{1}{2}, & & x \geqq 0 .
\end{aligned}
$$

Then $f, g \in C_{s}(X)$ but $f g \notin C_{s}(X)$. On the other hand, if $f \geqq 0$ and $h \geqq 0$ and if $f, h \in C_{s}(X)$, and if $B_{f} \cap B_{h} \neq \varnothing$, then $f h \in C_{s}(X)$. This observation will be useful in what follows.

2.2. Definition. A subspace $M$ of $X$ is a boundary for $C(X)$ if and only if for every $f \in C_{s}(X)$ we have $M \cap B_{f} \neq \varnothing$. If there is a smallest such subspace, we call it the minimal boundary of $C(X)$, and denote it by $M_{X}$.

When $X$ is compact this definition coincides with that given in [1]. It is to be noted that if $X$ is not pseudo-compact, there is no analogous concept of minimal boundary defined by all the continuous functions on $X$, since some of those are unbounded. However, it might be possible to consider a boundary defined by the larger class of continuous functions on $X$ which are bounded but which do not necessarily attain their maximum absolute values. We shall not develop this idea further here. We also mention that we could just as well consider complex-valued functions. The notion of minimal boundary would be the same, and all the theorems parallel to those given here.

It is obvious that $X$ itself is always a boundary, so that the notion of "smallest" always makes sense, even if such a set might not exist. However, it is perfectly conceivable that a "minimal" boundary might exist without it being the "smallest" boundary. Thus we need to justify the terminology.

First we reduce our study to topological spaces which are both completely regular and Hausdorff. For an arbitrary topological space $X$, the completely regular Hausdorff space $Y$ identified with $X$ is defined in [3, p. 41], and $C(X)$ 
and $C(Y)$ are isomorphic in every sense; so are $C_{s}(X)$ and $C_{s}(Y)$. In particular, if $x \in X$, let $x_{1}$ be the equivalence class consisting of all $y \in X$ such that for each $f \in C(X)$, we have $f(y)=f(x)$. Then the $Y$ defined above is in fact the set of all such equivalence classes, endowed with the topology inherited from $X$.

2.3. TheOREM. Let $X$ be a topological space and let $Y$ be the completely regular Hausdorff space derived above. Then $M_{X}$ exists if and only if $M_{Y}$ exists, and when they do exist they are identifiable.

Proof. Let $M_{X}$ exist, and let $M=\left\{x_{1}\right.$ : there exists an $x \in X$ such that $x \in x_{1}$ and $\left.x \in M_{X}\right\}$. Then certainly $M$ is a boundary for $C(Y)$. That it is the smallest boundary for $C(Y)$ follows from the fact that $M_{X}$ is the smallest boundary on $C(X)$ and from the construction of $Y$. So $M=M_{Y} ; M_{X}$ and $M_{Y}$ are identified in the obvious way. Now let $M_{Y}$ exist, and for each $x_{1} \in M_{Y}$ let $x_{0}$ be a fixed element of $x_{1}$. Let $M=\left\{x_{0} \in X: x_{1} \in M_{Y}\right\}$. By the isomorphism of $C_{s}(X)$ and $C_{s}(Y), M$ is a boundary for $X$, and the fact that $M_{Y}$ is the smallest boundary for $Y$ and the uniqueness of $x_{0}$ in each $x_{1}$ yields $M$ as the minimal boundary for $X$. Thus $M=M_{X}$. Once again $M_{X}$ and $M_{Y}$ can be identified in the natural way.

$N . B$. Henceforth, we shall always assume that any topological space we discuss is completely regular and Hausdorff.

Next we determine which spaces are endowed with a minimal boundary. For a subspace $D$ of $X$, let $X-D$ be the complement in $X$ of $D$.

2.4. Lemma. If $A$ is a nonvoid $G_{\delta}$ subset of $X$, then there exists an $f \in C_{s}(X)$ such that $B_{f} \subseteq A$.

Proof. Let $A=\bigcap_{n=1}^{\infty} U_{n}, U_{n}$ open in $X$, and let $x \in A$ be fixed. Then the complete regularity of $X$ yields a function $f_{n}: X \rightarrow\left[0,1 / 2^{n}\right]$ which is continuous, such that $f_{n}(x)=1 / 2^{n}$ and $f_{n}\left(X-U_{n}\right)=0$. Let $f=\sum_{n=1}^{\infty} f_{n}$. Then $f \in C_{s}(X)$ and $B_{j} \subseteq A$.

2.5. Corollary. If $\{x\}$ is a $G_{\delta}$, then $B_{f}=\{x\}$ for some $f \in C_{s}(X)$.

2.6. Lemma. If $M$ is a boundary for $C(X)$ and if $x$ is not a $G_{\delta}$ point, then $M-\{x\}$ is a boundary for $C(X)$.

Proof. Assume without loss of generality that $x \in M$, and let $f \in C_{s}(X), f \geqq 0$, such that $x \in B_{f}$. We must show that $(M-\{x\}) \cap B_{f} \neq \varnothing$. Inasmuch as $B_{f}$ is a closed $G_{\delta}\left[3\right.$, p. 15] and $x$ is assumed to be a non- $G_{\delta}$ point, there exists a point $z \in B_{f}$ such that $z \neq x$. The complete regularity of and Hausdorff topology on $X$ yield a continuous function $h: X \rightarrow[0,1]$ such that $h(z)=1$ and $h(x)=0$. Then $f+h \in C_{s}(X)$ and $B_{f+h} \subseteq B_{f}-\{x\}$. But $M$ is a boundary for $C(X)$, so $M \cap B_{f+h} \neq \varnothing$. Therefore we have $(M-\{x\}) \cap B_{f} \neq \varnothing$. Since the $f \in C_{s}(X)$ was arbitrary, the lemma follows.

2.7. THEOREM. For any space $X$, the minimal boundary $M_{X}$ exists if and only if every nonempty closed $G_{\delta}$ subset of $X$ contains a $G_{\delta}$ point. 
Proof. We first show that if $M_{X}$ exists, then every nonempty closed $G_{\delta}$ in $X$ contains a $G_{\delta}$ point. Assume that $A$ is a nonvoid closed $G_{\delta}$ without any $G_{\delta}$ points, and let $M$ be an arbitrary boundary for $C(X)$. By Lemma 2.4, there is an $f \in C_{s}(X)$ such that $B_{f} \subseteq A$. Then $M \cap B_{j} \neq \varnothing$, so that there is an $x \in M \cap B_{f} \subseteq A$. By hypothesis, $x$ is not a $G_{\delta}$, so Lemma 2.6 yields $M-\{x\}$ as a boundary. Therefore $M$ cannot be the smallest boundary. Conversely, assume the condition holds, and let $M=\left\{x \in X:\{x\}\right.$ is a $G_{\delta}$ point $\}$. Then $M$ is certainly a boundary because the maximum absolute value of any function in $C_{s}(X)$ is a closed $G_{\delta} ; M$ is the smallest boundary by virtue of Lemma 2.5 .

We note immediately that if $X$ is metrizable, then $M_{X}$ exists, since each element of $X$ is a $G_{\delta}$. In fact, the same is true if the first countability axiom prevails on $X$. That this is a strictly larger class of spaces is assured by the existence of a compact Hausdorff space for which the first countability axiom holds but which is not metrizable [6, p. 164]. A space $X$ for which $M_{X}$ exists and is different from $X$ is the ordinals less than or equal to the first uncountable $\Omega$, with the order topology. Then $M_{X}=X-\{\Omega\}$. Finally, if $X$ is a nonmetrizable topological group which is $T_{0}$, then $M_{X}$ does not exist [4, Theorem 8.3], and if $X$ is an uncountable product of nontrivial spaces with the product topology, then also $M_{X}$ does not exist. In each case there are no $G_{\delta}$ points in the space.

2.8. Corollary. If $M_{X}$ exists, then it consists precisely of the $G_{\delta}$ points in $X$.

2.9. Corollary. If $M_{X}$ exists, then it is dense in $X$.

Proof. Simply note the definition of complete regularity.

2.10. COROLlaRY. If $M$ is a boundary which contains properly no other boundary, then $M=M_{X}$.

Proof. If $M$ contains no other boundary, then it is precisely the collection of $G_{\delta}$ points, so $M=M_{X}$.

Corollary 2.10 tells us that the boundaries for $C(X)$ form a lattice, that the concept of minimal and of smallest boundary coincide, so the term "minimal boundary" is grammatically sound. It is easy to show that if $X$ is compact and Hausdorff, then the Silov boundary [9], Choquet boundary [8], and strong boundary [9] of $C(X)$ exist, are identical, and are equal to $X$, so that when the minimal boundary exists, it is dense in each of these boundaries. It is also apparent that if $X$ and $Y$ are homeomorphic, then $M_{X}$ and $M_{Y}$ are coexistent if either deigns to exist, and when they exist, they are homeomorphic. Nevertheless, if $X$ and $Y$ are given, and if $f: X \rightarrow Y$ is a one-to-one and onto and continuous map, and if $M_{X}$ exists, this in no way implies that $M_{Y}$ exists. Just let $Y$ be a space for which $M_{Y}$ does not exist, and let $X$ be the same space as a set, but with the discrete topology, and let $f$ be the identity function. Then $M_{X}=X$, and there is no $M_{Y}$.

A natural question to ask is if the process of obtaining minimal boundaries can continue; i.e. if $M_{X}$ is the minimal boundary for $C(X)$, does $M_{\left(M_{X}\right)}$ exist? If 
$M_{\left(M_{X}\right)}$ does exist, may $M_{\left(M_{X}\right)} \neq M_{X}$ ? Since each point in $M_{X}$ is a $G_{\delta}, M_{\left(M_{X}\right)}$ exists and $M_{\left(M_{X}\right)}=M_{X}$, so the process happily stops after the initial step.

A topological space $X$ is said to be dispersed if it contains no nonvoid perfect subspace [7]. For such spaces we have the following

2.11. THEOREM. If $X$ is dispersed, then $M_{X}$ exists.

Proof. Let $A$ be a nonvoid $G_{\delta}$ subset of $X$. Since any subspace of a dispersed space is also dispersed, so is $A$, and this means that there is an isolated point $x \in A$. Thus there is an open set $U$ in $X$ such that $U \cap A=\{x\}$. On the other hand, $A=\bigcap_{n=1}^{\infty} U_{n}$, where $U_{n}$ is open in $X$. This means that $\{x\}=\bigcap_{n=1}^{\infty}\left(U \cap U_{n}\right)$, so that $x$ is a $G_{\delta}$ point in $X$. Now refer to Theorem 2.7, and the proof is complete.

We have shown that dispersed spaces and metrizable spaces each have minimal boundaries. These types of spaces are different. For the one-point compactification of a discrete space is dispersed, and if uncountable, then it is not metrizable. The same is true for the space of ordinals less than or equal to the first uncountable. Next we look at spaces for which the minimal boundary does not exist. A space $X$ is called extremally disconnected if and only if the closure of each open set is open $[3$, p. 22].

2.12. THEOREM. If $X$ is an infinite compaci extremally disconnected space, then $M_{X}$ fails to exist.

Proof. Assume that $M_{X}$ exists. Then each point of $M_{X}$ is a $G_{\delta}$, which means rather more-namely, that each point of $M_{X}$ is isolated. Since $M_{X}$ is dense in $X$ which is infinite, $M_{X}$ contains an infinite set $\left(x_{n}\right)_{n=1}^{\infty}$. Define $f: M_{X} \rightarrow[0,1)$ by $f\left(x_{n}\right)=1-1 / n$, all $n$, and $f(x)=0$, for all other $x \in M_{X}$. Then $f$ is continuous on $M_{X}$, and has a continuous extension to $X\left[3\right.$, p. 96]. Then $B_{f} \cap M_{X}=\varnothing$, so that $M_{X}$ cannot be a boundary.

The theorem is not true for all extremally disconnected spaces, since if $X$ is discrete, then it is extremally disconnected but $M_{X}=X$. On the other hand, there are numerous infinite compact extremally disconnected spaces. Let $Y$ be an infinite compact (Hausdorff) space, and let $C^{* *}(Y)$ be the second conjugate space of $C(Y)$. Then $C^{* *}(Y)$ is isometrically (and algebraically!) isomorphic to $C(X)$, where $X$ is an appropriate compact, extremally disconnected space [5, p. 1022]. Furthermore, $Y$ is embedded as a dense discrete space in $X$. Consequently, even if $X$ has a dense subspace of isolated points, $M_{X}$ need not exist.

It is clear that if $M_{X}$ exists, it need not be open in $X$. For let $Y$ be such that $M_{Y}$ exist but $M_{Y} \neq Y$, and for each $n$, let $Y_{n}=Y$, and set $X=\prod_{n=1}^{\infty} Y_{n}$, where $X$ is endowed with the product topology. Then $M_{X}$ exists and $M_{X}=\prod_{n=1}^{\infty} M_{Y_{n}}$ (this will follow directly from Theorem 3.5). However, things are worse. If we consider the smallest $\sigma$-algebra generated by the closed and open sets of $X$ to be the Borel sets of $X$, then our next example shows that $M_{X}$ can exist without being a Borel set. 
2.13. ExAmple. Let $Y=[0,1]$ with the usual topology, and let $Z$ be a non-Borel subset of $Y$, which by the axiom of choice exists. Let the elements of $Z$ be denoted by $\left\{y_{\lambda}: \lambda \in \Lambda\right\}, \Lambda$ an index set. For each $\lambda \in \Lambda$, let $Y_{\lambda}$ be the collection of ordinals less than or equal to the first uncountable under the order topology, and let the first uncountable ordinal be $y_{\lambda}$ itself. Think of the $Y_{\lambda}$ as being distinct entities. Let $X=Y \cup \bigcup_{\lambda \in \Lambda} Y_{\lambda}$. For $x \in X$ such that $x \in Y_{\lambda}$ for some $\lambda \in \Lambda$, and such that $x \neq y_{\lambda}$, let a neighborhood basis consist of those sets in $Y_{\lambda}$ which are neighborhoods of $x$ in the order topology of $Y_{\lambda}$. For $x \in Y$, let a neighborhood $V$ of $x$ contain a neighborhood $U$ of $x$ in $[0,1]$ (with its own usual topology) together with a neighborhood in the order topology of $Y_{\lambda}$ for each $y_{\lambda}$ in $U$. It is simple to verify that $X$ is a topological space and is Hausdorff. Furthermore, $Y$ and each $Y_{\lambda}$ are embedded topologically in $X$. To show that $X$ is completely regular, let $A$ be a nonvoid closed subset of $X$, and $x \notin A$. If $x \notin Y$, then there is a $\lambda \in \Lambda$ such that $x \in Y_{\lambda}$. Since $Y_{\lambda}$ is completely regular, there is a continuous function $f_{0}: Y_{\lambda} \rightarrow[0,1]$ such that $f_{0}(y)=0$ for $y \in Y_{\lambda}$ with $y>x$, and $f_{0}(x)=1$, and $f_{0}\left(A \cap Y_{\lambda}\right)=0$. Now define $f: X \rightarrow[0,1]$ by $f(y)=f_{0}(y)$, for all $y \in Y_{\lambda}$, and $f(y)=0$, all other $y \in X$. Then $f$ is continuous on $X$, and $f(x)=1$, while $f(A)=0$. Next, if $x \in Y$, then there is an open set $U$ in $Y$ with $x \in U$, and with the property that for each $y_{\lambda}$ in $U$ there is an open set $V_{\lambda}$ in $Y_{\lambda}$, with $y_{\lambda} \in V_{\lambda}$, and such that $\left(U \cup \bigcup_{y_{\lambda} \in U} V_{\lambda}\right) \cap A=\varnothing$. Since $Y$ is completely regular, there is a continuous function $f_{0}: Y \rightarrow[0,1]$ such that $f_{0}(x)=1$ while $f_{0}(Y-U)=0$. Furthermore, for each $\lambda \in \Lambda$, there is a continuous function $f_{\lambda}: Y_{\lambda} \rightarrow\left[0, f_{0}\left(y_{\lambda}\right)\right]$ such that $f_{\lambda}\left(y_{\lambda}\right)$ $=f_{0}\left(y_{\lambda}\right)$ and $f_{\lambda}\left(Y-V_{\lambda}\right)=0$. Now define $f: X \rightarrow[0,1]$ by $f(y)=f_{0}(y)$, for all $y \in Y$, and $f(y)=f_{\lambda}(y)$, for all $y \in Y_{\lambda}$, for each $\lambda \in \Lambda$. Then $f$ is continuous on $X$, and $f(x)=1$, and $f(A)=0$, which is just what we want. Thus $X$ is completely regular.

Next we identify the $G_{\delta}$ points in $X$. Certainly if $x \in Y_{\lambda}$ and $x \neq y_{\lambda}$, then $x$ is a $G_{\delta}$ point in $Y_{\lambda}$, and hence in $X$. If $x \in Y$ with $x \neq y_{\lambda}$, for all $\lambda \in \Lambda$, then let $\left(U_{n}\right)_{n=1}^{\infty}$ be an open neighborhood system for $x$ in $Y$, and let $V_{n}=U_{n} \cup \bigcup_{\lambda \in \Lambda_{n}} Y_{\lambda}$, where $\Lambda_{n}$ consists of all $\lambda \in \Lambda$ such that $y_{\lambda} \in U_{n}$. Then $V_{n}$ is open in $X$, and $\bigcap_{n=1}^{\infty} V_{n}=\{x\}$, so that $x$ is a $G_{\delta}$ in $X$. Finally, if $x=y_{\lambda}$ for some $\lambda \in \Lambda$, then $x$ is not a $G_{\delta}$ in $X$, since it is not a $G_{\delta}$ in $Y_{\lambda}$, which is topologically embedded in $X$. Hence the $G_{\delta}$ points in $X$ consist of the set $X-Z$. However, if $C$ is a nonvoid closed $G_{\delta}$ set in $X$, and if $y_{\lambda} \in C$, then there exists $y \in Y_{\lambda}$, with $y \neq y_{\lambda}$, such that $y \in C$. But such a $y$ is, by what we have just said, a $G_{\delta}$ in $X$. Thus $C$ contains a $G_{\delta}$ point. Therefore $M_{X}$ exists, and $M_{X}=X-Z$. Note that if $X-Z$ were in fact a Borel set in $X$, then $Z$ would also be Borel in $X$, so that $Z=Z \cap Y$ would be Borel in $Y$, which by assumption it is not. Thus $M_{X}$ is not a Borel subset of $X$.

We also have an example of a space $X$ such that not only $M_{X}$ exists and is dense in $X$, but also $X-M_{X}$ is dense in $X$. Ours is too complicated to print here. Obviously no point can be isolated. The example reminds one of the subject of study in the "corona problem" [10]. 
3. Inheritance properties of the minimal boundary. Suppose that for a given space $X, M_{X}$ exists, and let $Y$ be a closed subspace of $X$. Does $M_{Y}$ exist? And if $M_{Y}$ does exist, does $M_{Y}=M_{X} \cap Y$ ? In this section we answer these questions. We conclude by giving a criterion for products to have minimal boundaries.

3.1. THEOREM. Let $Y$ be an arbitrary space. Then there exists a (completely regular Hausdorff) space $X$ with the following properties:

1. $Y \subseteq X$ (homeomorphically).

2. $M_{X}$ exists.

3. If $M_{Y}$ exists, then $M_{Y}=M_{X} \cap Y$.

Proof. Let $Z=\left(z_{\lambda}\right)_{\lambda \in \Lambda}$, where $\Lambda$ is an uncountable index set and $0 \in \Lambda$, and let $Z$ have the discrete topology. Let $X=Y \times Z$, and define the following as neighborhoods in $X$, for all $(y, z) \in X$ :

1. If $z \neq z_{0}$, then $\{(y, z)\}$ is a neighborhood of itself.

2. If $z=z_{0}$, then $W$ is a neighborhood of $\left(y, z_{0}\right)$ if $W \supseteq U$, where $U=(V-\{y\}, Z)$ $\cup\left\{\left(y, z_{0}\right)\right\}$ for some neighborhood $V$ of $y$ in $Y$.

In order to show that we have described a topology on $X$, we may show that $U$ is a neighborhood of each of its points, for $U$ a neighborhood of $\left(y_{0}, z_{0}\right)$. If $(y, z) \in U$ and $z \neq z_{0}$, then by $1, U$ is a neighborhood of $(y, z)$. If $y \neq y_{0}$ but $z=z_{0}$, then since $Y$ is assumed to be Hausdorff, there is a neighborhood $V_{1}$ of $y$ contained in $V$ such that $y_{0} \notin V_{1}$. But then $\left(V_{1}, Z\right) \subseteq U$ and by the definition in $2,(V, Z)$ is a neighborhood of $(y, z)$. Thus we have a topology on $X$. Since $Y$ is Hausdorff and $Z$ has the discrete topology, $X$ is Hausdorff. It is straightforward to show that $X$ is completely regular, and we omit the proof.

Next we remark that $\left(Y, z_{0}\right)$ is homeomorphic to $Y$. For $U$ is a neighborhood of $\left(y, z_{0}\right)$ if and only if $\left[U \cap\left(Y, z_{0}\right)\right] \supseteq\left(V, z_{0}\right)$ for some neighborhood $V$ of $y$ in $Y$. Thus $Y$ is embedded topologically in $X$. Toward showing that $M_{X}$ exists, we note that if $z \neq z_{0}$, then $\left(y, z_{0}\right)$ is a $G_{\delta}$, for all $y \in Y$. Next, if $y$ is a $G_{\delta}$ in $Y$ and if $\bigcap_{n=1}^{\infty} V_{n}=\{y\}$, with $V_{n}$ open in $Y$, then $\bigcap_{n=1}^{\infty}\left[\left(V_{n}-\{y\}, Z\right) \cup\left(y, z_{0}\right)\right]=\left\{\left(y, z_{0}\right)\right\}$, so that $\left(y, z_{0}\right)$ is a $G_{\delta}$ in $X$. Now if $y$ is not a $G_{\delta}$ in $Y$, then for any countable collection $\left(V_{n}\right)_{n=1}^{\infty}$ of neighborhoods for $y$ in $Y$, there is $y_{1} \in \bigcap_{n=1}^{\infty} V_{n}$, whence $\left(y_{1}, Z-\left\{z_{0}\right\}\right)$ $\subseteq \bigcap_{n=1}^{\infty}\left(V_{n}-\{y\}, Z\right) \cup\left(y, z_{0}\right)$, which itself is an arbitrary $G_{\delta}$ in $X$ containing $\left(y, z_{0}\right)$. However, every element of $\left(y_{1}, Z-\left\{z_{0}\right\}\right)$ is discrete, so that any (not necessarily closed) nonvoid $G_{\delta}$ in $X$ which contains $\left(y, z_{0}\right)$ also contains a $G_{\delta}$ point. Thus $M_{X}$ exists. From our proof we see that if $M_{Y}$ also exists, then $M_{X} \cap Y=M_{Y}$.

It is interesting to observe that $X$ is never compact. To prove it, let $y_{0} \in Y$ and let $U=\left(Y-\left\{y_{0}\right\}, Z\right) \cup\left(y_{0}, z_{0}\right)$, so that $U$ is open in $X$. But then $X-U=$ $\left(y_{0}, Z-\left\{z_{0}\right\}\right)$, which is an uncountable discrete set, so that $X$ cannot possibly be compact. The same reasoning shows that $X$ is not even locally compact.

Inasmuch as $Y$ is embedded as a closed subspace of $X$, Theorem 3.1 gives the answer "not always" to the first question at the beginning of this section. However, 
Theorem 3.1 does not settle the second question. Trivially the answer to it is again "not always." For let $X$ be such that $M_{X}$ exists and $M_{X} \neq X$, and let $y \in X-M_{X}$ and $Y=\{y\}$. Then $M_{Y}=\{y\}$ and $M_{X} \cap Y=\varnothing$. Perhaps this is not a satisfying answer. A more fruitful settlement of the question is contained in

3.2. THEOREM. Let $Y$ be an arbitrary space. Then there exists a compact Hausdorff space with the following properties:

1. $Y \subseteq X$ (homeomorphically).

2. $M_{X}$ exists.

3. $M_{X} \cap Y=\varnothing$.

Proof. For the time being, assume that $Y$ is compact. Define $X=Y \times Z$ as a set, just as in Theorem 3.1. Let the following be neighborhoods in $X$, for the arbitrary element $(y, z)$ :

1. If $z \neq z_{0}$, then $\{(y, z)\}$ is a neighborhood of itself.

2. If $z=z_{0}$, then $W$ is a neighborhood of $\left(y, z_{0}\right)$ if $W \supseteq U$, where

$$
W=\left[(V, Z \text {-finite subset of } X] \cup\left\{\left(y, z_{0}\right)\right\},\right.
$$

for some neighborhood $V$ of $y$ in $Y$.

Indeed this describes a topology on $X$, as is easy to check, and once again, since $Y$ is Hausdorff and $Z$ has the discrete topology, $X$ is Hausdorff too. Now we show that $X$ is compact-whence completely regular. Let $\left(W_{\gamma}\right)_{y \in \Gamma}$ be an open cover of $X$. For each $\left(y, z_{0}\right)$ in $X$, there is an open neighborhood $U_{y}$ of $y$ in $Y$ such that $\left(y, z_{0}\right) \in\left[\left(U_{y}, Z\right)\right.$-finite set $] \subseteq W_{y}$, for some $\gamma \in \Gamma$ (depending of course on the chosen $y$ ). Since $Y$ is assumed to be compact, there are $y_{1}, \ldots, y_{n}$, such that $\bigcup_{k=1}^{n} U_{y_{k}}$ covers $Y$. But then $X-\bigcup_{k=1}^{n}\left[\left(U_{y_{k}}, Z\right)\right.$ - finite set $]$ is a finite point set. Therefore $X$ can be covered by a finite subcollection of $\left(W_{y}\right)_{y \in \Gamma}$. This means $X$ is compact.

Now we show that $M_{X}$ exists. If $z \neq z_{0}$, then $(y, z)$ is isolated, so is a $G_{\delta}$ point. Next, let $\left(y, z_{0}\right)$ be an element of any $G_{\delta}$ set $\bigcap_{n=1}^{\infty} W_{n}$, where each $W_{n}$ is open in $X$. Then there are $V_{n}, n=1,2, \ldots$, open in $Y$, such that $\left(y, z_{0}\right) \in[(y, Z)$-countable set $]$ $\cup\left\{\left(y, z_{0}\right)\right\} \subseteq \bigcap_{n=1}^{\infty}\left[\left(V_{n}, Z\right)\right.$ - countable set $] \subseteq \bigcap_{n=1}^{\infty} W_{n}$. But $[(y, Z)$-countable set] contains an uncountable collection of isolated points. Thus $\bigcap_{n=1}^{\infty} W_{n}$ contains $G_{\delta}$ points. It is then apparent that $M_{X}$ exists. With a simple calculation we find that $Y$ is homeomorphic to $\left(Y, z_{0}\right)$; by construction $\left(Y, z_{0}\right)$ has no $G_{\delta}$ points in $X$. Thus $M_{X} \cap Y=\varnothing$-provided that $Y$ is compact. Now let $Y$ be an arbitrary completely regular Hausdorff space, and let $\beta Y$ be the Stone-Čech compactification of $Y$. Finally, let $X=\beta Y \times Z$, and put the topology defined above on $X$, with respect to $\beta Y$ and $Z$. Then $X$ is compact and $M_{X}$ exists. Furthermore, $\beta Y$ is homeomorphic to $\left(\beta Y, z_{0}\right)$, so that $Y$ is homeomorphic to $\left(Y, z_{0}\right)$, with the result that $Y$ is embedded topologically in $X$. Once again $M_{X} \cap Y=\varnothing$, and the proof is complete.

Although Theorems 3.1 and 3.2 give a negative answer to the questions at the 
outset of this section, we now show that under certain circumstances the answers to both questions are yes.

3.3. Lemma. If $Y$ is a subspace of $X$, and if both $M_{X}$ and $M_{Y}$ exist, then $M_{Y} \supseteq M_{X} \cap Y$.

Proof. This follows immediately from Corollary 2.8 and from the observation that a point $G_{\delta}$ in $X$ which is in $Y$ is a point $G_{\delta}$ in $Y$.

3.4. THEOREM. Let $X$ be an arbitrary space, and $Y$ a nonvoid $G_{\delta}$ in $X$. If $M_{X}$ exists, then $M_{Y}$ exists and $M_{Y}=M_{X} \cap Y$.

Proof. If $A$ is a nonvoid closed $G_{\delta}$ in $Y$, then $A$ is at least a $G_{\delta}$ in $X$, so that by Lemma 2.4, there is an $f \in C_{s}(X)$ such that $B_{f} \subseteq A$. By hypothesis and by Theorem 2.7, $B_{f}$ contains a $G_{\delta}$ point $y($ in $X)$. Then $y$ is a $G_{\delta}$ in $Y$, and therefore $M_{Y}$ exists. Since $G_{\delta}$ points in the $G_{\delta}$ subspace $Y$ are $G_{\delta}$ points in $X, M_{Y}=M_{X} \cap Y$.

Theorem 3.4 tells us that some of the closed subspaces-at least those which are closed $G_{\delta}$ sets-inherit minimal boundaries. So also for all open subspaces. In the final theorem of this section, we characterize those product spaces which have minimal boundaries, in terms of their coordinate spaces.

3.5. THEOREM. Let $\Lambda$ be an index set, and for each $\lambda \in \Lambda$, let $Y_{\lambda}$ be a nontrivial topological space. Let $X=\prod_{\lambda \in \Lambda} Y_{\lambda}$, endowed with the product topology. Then $X$ has a minimal boundary if and only if $\Lambda$ is at most countable and for each $\lambda \in \Lambda, Y_{\lambda}$ has a minimal boundary. If $X$ has a minimal boundary, then $M_{X}=\prod_{\lambda \in \Lambda} M_{Y_{\lambda}}$.

Proof. If $\Lambda$ is uncountable, then no points of the product space $X$ are $G_{\delta}$ points, so that there can be no minimal boundary. Next, if $\Lambda$ is at most countable, but some coordinate space, say $Y_{1}$, has no minimal boundary, then there is a nonvoid closed $G_{\delta}$ set $A_{1}$ in $Y_{1}$ without any $G_{\delta}$ points. Let $A_{k}=Y_{k}$ for all $k \neq 1$, and let $A=\prod_{k \in \Lambda} A_{k}$. Then $A$ is a nonvoid closed $G_{\delta}$ in $X$. Since the projection of a $G_{\delta}$ point in $X$ onto any of its coordinates must be a $G_{\delta}$ point in the coordinate space, we find that $A$ has no $G_{\delta}$ points in $X$. Thus in this case $M_{X}$ fails to exist. We have remaining the case in which $\Lambda$ is at most countable and each $Y_{k}$ has a minimal boundary. We will show that $M_{X}$ exists and $M_{X}=\prod_{k \in \Lambda} M_{Y_{k}}$. A routine check shows that if for each $k, y_{k}$ is a $G_{\delta}$ in $Y_{k}$, then $x=\left(y_{k}\right)_{k \in \Lambda}$ is a $G_{\delta}$ in $X$. Now let $A$ be a nonvoid closed $G_{\delta}$ in $X$. We will find a $G_{\delta}$ point $x$ in $A$. To this end, note that $A=\bigcap_{n=1}^{\infty} W_{n}$, where $W_{n}=\bigcup_{i=1}^{m_{n}} \prod_{k \in \Lambda} W_{n, k, i}$, and where each $W_{n, k, i}$ is at least open in $Y_{k}$. Fix $y=\left(y_{k}\right)_{k \in \Lambda}$ in $A$. Then for each $n$, there exists $i_{n}$ with $i_{n} \leqq m_{n}$, such that $\prod_{k \in \Lambda} W_{n, k, i_{n}}$ contains $y$. But for each $n$ and $k$, there is a continuous function $f_{n k}$ such that $f_{n k}: Y_{k} \rightarrow\left[0,1 / 2^{n}\right], f_{n k}\left(y_{k}\right)=1 / 2^{n}$ and $f_{n k}\left(Y_{k}-W_{n, k, i_{n}}\right)=0$. Define $f_{k}=\sum_{n=1}^{\infty} f_{n k}$, so that $f_{k} \in C_{s}\left(Y_{k}\right)$ and $B_{f_{k}} \subseteq \bigcap_{n=1}^{\infty} W_{n, k, i_{n}}$. By hypothesis, there is a $G_{\delta}$ point $x_{k}$ in $B_{f_{k}}$, for each $k$. Let $x=\left(x_{k}\right)_{k \in \Lambda}$. Then by our remark above, $x$ is a $G_{\delta}$ in $X$, and $x \in \prod_{k \in \Lambda} B_{f_{k}} \subseteq \bigcap_{n=1}^{\infty} \prod_{k \in \Lambda} W_{n, k, i_{n}} \subseteq \bigcap_{n=1}^{\infty} W_{n}=A$. Therefore $M_{X}$ exists. Evidently $M_{X}=\prod_{k \in \Lambda} M_{Y_{k}}$. 


\section{Minimal boundaries for compactifications.}

4.1. THEOREM. Let $X_{0}$ be a locally compact, noncompact Hausdorff space, and let $X$ be the one-point $\left(x_{\infty}\right)$ compactification of $X_{0}$. Then $M_{X}$ exists if and only if $M_{X_{0}}$ exists, and if they exist, then $M_{X}=M_{X_{0}} \cup\left\{x_{\infty}\right\}$ if and only if $X$ is $\sigma$-compact.

Proof. Since $X$ is Hausdorff, $X_{0}$ is open in $X$, so we can invoke Theorem 3.4 to show that if $M_{X}$ exists, then $M_{X_{0}}$ exists. Now we prove the reverse implication. First we note that if $x$ is a $G_{\delta}$ point in $X_{0}$, then it is a $G_{\delta}$ in $X$ since $X_{0}$ is open in $X$. Assume that $M_{X_{0}}$ exists, and let $A$ be a nonvoid closed $G_{\delta}$ subset of $X$. Then $A_{0}=A \cap X_{0}$ is a closed $G_{\delta}$ in $X_{0}$. If $A_{0}=\varnothing$, then $A=\left\{x_{\infty}\right\}$, which is thus a $G_{\delta}$ point in $X$. Otherwise $A_{0} \neq \varnothing$, and by the hypothesis, there is a $G_{\delta}$ point $y$ in $A_{0}$. Then $y$ is a $G_{\delta}$ point in $X$, with the result that $M_{X}$ exists. By the definition of the topology on one-point compactification, $x_{\infty}$ is a $G_{\delta}$ point if and only if $X$ is $\sigma$-compact, so that the last statement of the theorem is a consequence of Corollary 2.8 .

We mention that if $X_{0}$ is the space of ordinals less than the first uncountable, with the order topology, then $X$ is the space of ordinals less than or equal to the first uncountable, and $M_{X}=X_{0}=M_{X_{0}}$, while if $X_{0}=(0,1]$ with the usual topology, then $X=[0,1]$, and $M_{X}=[0,1] \neq(0,1]=M_{X_{0}}$.

Next we turn to the Stone-Čech compactification $\beta X$ of an arbitrary completely regular Hausdorff space $X$. This compactification always exists for such $X[3$, p. 86].

4.2. Lemma. Let $X$ be pseudo-compact. Then $x$ is $a G_{\delta}$ in $X$ if and only if $x$ is a $G_{\delta}$ in $\beta X$.

Proof. If $x \in \beta X$ and is a $G_{\delta}$, then $x \in X$, since $\beta X-X$ has no $G_{\delta}$ points [3, p. 132]. But this means that $x$ is a $G_{\delta}$ in $X$. On the other hand, if $x \in X$ is a $G_{\delta}$, then surely $x$ is a $G_{\delta}$ in $\beta X$, since otherwise the Hausdorff topology on $\beta X$ would yield a function $f \in C_{s}(\beta X)$ such that $B_{f} \cap X=\varnothing$. But since $X$ is pseudocompact, this is impossible.

4.3. TheOREM. Assume that $X$ is not compact. We have the following:

1. If $X$ is not pseudo-compact, then $M_{\beta X}$ does not exist.

2. If $X$ is pseudo-compact, then $M_{\beta X}$ exists if and only if $M_{X}$ exists, and in this case, $M_{\beta X}=M_{X}$.

Proof. 1. If $X$ is not pseudo-compact, then there is an unbounded function $h$ on $X$ such that $h$ is continuous. Let $g=\max (1, h), f=1 / g$, so that $f: X \rightarrow(0,1]$ is continuous, and $[f(X)]^{-}=[0,1]$. Let $f_{1}$ be the continuous extension of $f$ to $\beta X$. Then $\left(1-f_{1}\right) \in C_{s}(\beta X)$ and $B_{1-f_{1}} \cap X=\varnothing$. However, no point in $\beta X-X$ can ever be a $G_{\delta}$. Thus the closed nonempty $G_{\delta}$ set $B_{1-f_{1}}$ in $\beta X$ has no $G_{\delta}$ point, and $\beta X$ therefore has no minimal boundary.

2. Assume that $M_{X}$ exists, and let $A$ be a nonvoid closed $G_{\delta}$ in $\beta X$. Then $A \cap X$ 
is a nonvoid closed $G_{\delta}$ in $X$, so by hypothesis contains a point which is a $G_{\delta}$ in $X$. But such points are $G_{\delta}$ in $\beta X$, by Lemma 4.2. Hence $\beta X$ has a minimal boundary. Conversely, assume that $M_{\beta X}$ exists, and let $A$ be a nonvoid closed $G_{\delta}$ in $X$. By Lemma 2.4 there is a function $f \in C_{s}(X)$ such that $B_{f} \subseteq A$. If $f_{1}$ is its continuous extension to $\beta X$, then $B_{f_{1}} \cap X \subseteq A$, and by hypothesis there exists a $G_{\delta}$ point $x$ in $B_{f_{1}}$. However, $x$ cannot be in $\beta X-X$ since $x$ is a $G_{\delta}$, so that $x \in A$ and is a $G_{\delta}$ in $X$. Therefore $M_{f}$ exists. Furthermore, since $\beta X-X$ has no $G_{\delta}$ points, Corollary 2.8 provides us with the final equality $M_{\beta X}=M_{X}$.

One of the consequences of Theorem 4.3 is that if $N, Q$, and $R$ are the spaces of integers, rationals, and reals, respectively, under their ordinary topologies, then $M_{\beta N}, M_{\beta Q}$, and $M_{\beta R}$ do not exist, although $M_{N}=N, M_{Q}=Q$, and $M_{k}=R$.

For an arbitrary space $Y$, let $C_{b}(Y)$ consists of all real-valued bounded continuous functions on $Y$, with supremum norm and pointwise multiplication. Henceforth assume that $X$ is compact and that $M_{X}$ exists. We ask under what conditions each function in $C_{b}\left(M_{X}\right)$ has a unique continuous extension to $X$. In other words, when are $C_{b}\left(M_{X}\right)$ and $C(X)$ naturally identifiable?

4.4. THEOREM. The following are equivalent:

1. $C_{b}\left(M_{X}\right)$ is identifiable with $C(X)$.

2. $M_{X}$ is pseudo-compact and $\beta M_{X}=X$.

3. $M_{X}$ is pseudo-compact and $f \in C(M X)$ is uniformly continuous with respect to the uniform structure on $X$ restricted to $M_{X}$.

Proof. If $\beta M_{X} \neq X$, then by the Stone-Čech compactification theorem, $C_{b}\left(M_{X}\right)$ is not identifiable with $C(X)$. Furthermore, if $C_{b}\left(M_{X}\right)$ and $C(X)$ are identifiable, then [2, Theorem IV.6.26] shows that $X$ and $\beta M_{X}$ are homeomorphic, so that $M_{\beta M_{X}}$ exists. But then Theorem 4.3 tells us that $M_{X}$ is pseudo-compact. Hence 1 implies 2. The converse is true by the Stone-Čech compactification theorem. Next we show that 2 implies 3 . If $f \in C_{b}\left(M_{X}\right)=C\left(M_{X}\right)$, then let $f_{0}$ be the continuous extension of $f$ to $\beta M_{X}=X$. Since $X$ is compact, $f_{0}$ is uniformly continuous on $X$, so $f$ is uniformly continuous on the restricted uniformity on $M_{X}$. Hence 2 implies 3. Conversely, assume that 3 holds, and let $f \in C\left(M_{X}\right)$. Then $f$ is uniformly continuous with respect to the given uniform structure on $M_{X}$, so has a unique continuous extension $f_{0}$ to the compact space $X$ by [6, Theorem 6.26]. But this is tantamount to the statement that $X=\beta M_{X}$. Thus 3 implies 2 .

Let us note that there is at least one nontrivial example which satisfies the conditions of Theorem 4.4: if $X$ is the space of ordinals less than or equal to the first uncountable, with its order topology, then $\beta M_{X}=X$ and $M_{X}$ is pseudo-compact, and $M_{X} \neq X$, and $C_{b}\left(M_{X}\right)$ is identifiable with $C(X)$. However, $X$ may be such that $M_{X}$ is pseudo-compact without $C_{b}\left(M_{X}\right)$ and $C(X)$ being identifiable. To create such a space, we let $Y$ and $Z$ each be distinct copies of the ordinals less than or equal to the first uncountable, endowed with the order topology, and let the first uncountables be denoted by $y_{0}$ and $z_{0}$ in $Y$ and $Z$ respectively. Assume that $y_{0}=z_{0}$. 
Let $X=Y \cup Z$, with neighborhoods of any $y<y_{0}$ and any $z<z_{0}$ just the neighborhoods they normally have in $Y$ and $Z$ respectively. Let the neighborhoods of $y_{0}=z_{0}$ be the union of neighborhoods of $y_{0}$ in $Y$ and $z_{0}$ in $Z$. Then $M_{X}=X-\left\{y_{0}\right\}$ $=X-\left\{z_{0}\right\}$, and $M_{X}$ inherits its pseudo-compactness from $Y$ and $Z$. However, $\beta M_{X} \neq X$. For take $f: M_{X} \rightarrow[0,1]$ defined by $f\left(Y-\left\{y_{0}\right\}\right)=0$ and $f\left(Z-\left\{z_{0}\right\}\right)=1$. Then $f$ is continuous on $M_{X}$ and has no continuous extension to $X$, so that $\beta M_{X} \neq X$. However, $\beta M_{X}$ is the two-point compactification of $\left(Y-\left\{y_{0}\right\}\right) \cup\left(Z-\left\{z_{0}\right\}\right)$. By [2, Theorem IV.6.26], $\beta M_{X}$ and $X$ are not homeomorphic; therefore $C_{b}\left(M_{X}\right)$ and $C(X)$ are not identifiable.

\section{REFERENCES}

1. E. Bishop, A minimal boundary for function algebras, Pacific J. Math. 9 (1959), 629-642.

2. N. Dunford and J. T. Schwartz, Linear operators, Part I, Interscience, New York, 1958.

3. L. Gillman and M. Jerison, Rings of continuous functions, Van Nostrand, Princeton, N. J., 1960.

4. E. Hewitt and K. A. Ross, Abstract harmonic analysis. Part I, Springer, Berlin, 1963.

5. S. Kakutani, Concrete representation of abstract (M)-spaces, Ann. of Math. 42 (1941), 994-1024.

6. J. L. Kelley, General topology, Van Nostrand, Princeton, N. J., 1955.

7. A. Pelczyński and Z. Semadeni, Spaces of continuous functions (III), Studia Math. 18 (1959), 211-222.

8. R. R. Phelps, Lectures on Choquet's theorem, Van Nostrand, Princeton, N. J., 1966.

9. C. E. Rickart, General theory of Banach algebras, Van Nostrand, Princeton, N. J., 1960.

10. I. J. Schark, Maximal ideals in an algebra of bounded analytic functions, J. Math. Mech. 10 (1961), 735-746.

UNIVERSTTY OF MARYLAND,

College Park, Maryland 